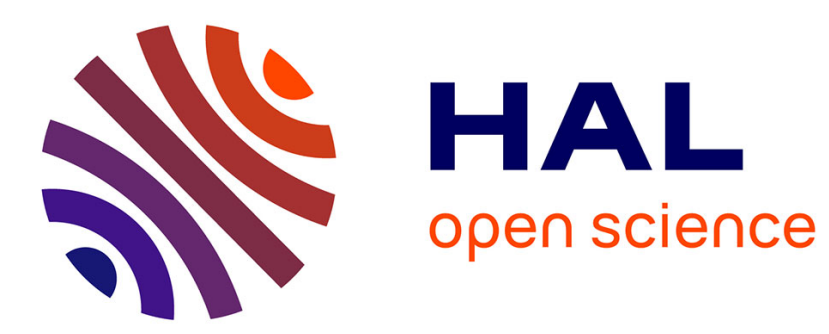

\title{
Le dépôt électrolytique de chrome noir : Caractérisation et stabilité thermique
}

J. Valignat, J. Spitz, I.T. Ritchie

\section{To cite this version:}

J. Valignat, J. Spitz, I.T. Ritchie. Le dépôt électrolytique de chrome noir: Caractérisation et stabilité thermique. Revue de Physique Appliquée, 1980, 15 (3), pp.397-402. 10.1051/rphysap:01980001503039700 . jpa-00244743

HAL Id: jpa-00244743

https://hal.science/jpa-00244743

Submitted on 1 Jan 1980

HAL is a multi-disciplinary open access archive for the deposit and dissemination of scientific research documents, whether they are published or not. The documents may come from teaching and research institutions in France or abroad, or from public or private research centers.
L'archive ouverte pluridisciplinaire $\mathbf{H A L}$, est destinée au dépôt et à la diffusion de documents scientifiques de niveau recherche, publiés ou non, émanant des établissements d'enseignement et de recherche français ou étrangers, des laboratoires publics ou privés. 


\title{
Le dépôt électrolytique de chrome noir : Caractérisation et stabilité thermique
}

\author{
J. Valignat, J. Spitz et I. T. Ritchie \\ Département de Métallurgie de Grenoble, Laboratoire d'Etudes des Matériaux Minces, \\ 38041 Grenoble Cedex, France
}

(Reçu le 3 octobre 1979, révisé le 12 décembre 1979, accepté le 14 décembre 1979)

\begin{abstract}
Résumé. - On décrit un procédé industriel de dépôt électrolytique de chrome noir, puis à l'aide de moyens physiques de caractérisation on étudie le mécanisme de la dégradation thermique des couches obtenues. I'n modèle permet de calculer les spectres de réflexion et de montrer que c'est la modification de la forme des particules au cours du recuit qui est le facteur prépondérant du changement irréversible des propriétés optiques.
\end{abstract}

\begin{abstract}
An industrial process of chromium black deposition is described and the obtained coatings are characterized before and after annealing. A model is described which enables the reflectance spectra to be reproduced.

This model shows that the modification in the shape of the particles in the coating is responsible for the irreversible changes of the optical properties which occur on annealing.
\end{abstract}

Introduction. - Depuis les premiers travaux de Tabor [1] en 1956, l'emploi de surfaces sélectives pour la conversion photothermique de l'énergie solaire s'est considérablement développé. En effet, dans de nombreuses applications, il est possible de diminuer notablement les surfaces de captation en remplaçant des absorbeurs noirs par des absorbeurs sélectifs.

Un tel absorbeur est caractérisé par le fait qu'il absorbe la quasi-totalité du rayonnement solaire incident comme le ferait un corps noir, mais à la différence de ce dernier, il n'émet que très peu de radiations dans l'infrarouge (Fig. 1). Son rendement de conversion se trouve donc sensiblement augmenté du fait de la réduction des pertes radiatives, qui sont proportionnelles à la puissance quatrième de la température.

Différents phénomènes permettent de réaliser des surfaces sélectives $[2,3,4]$ :

- utilisation d'un matériau ayant des propriétés sélectives intrinsèques;

- utilisation d'un filtre interférentiel réalisé par l'empilement de couches alternées de deux matériaux ayant des indices optiques différents (en général un métal et un diélectrique);

- utilisation des effets de diffusion de la lumière, soit dans le volume, soit à la surface du matériau;

- utilisation d'un empilement absorbeur plus réflecteur.

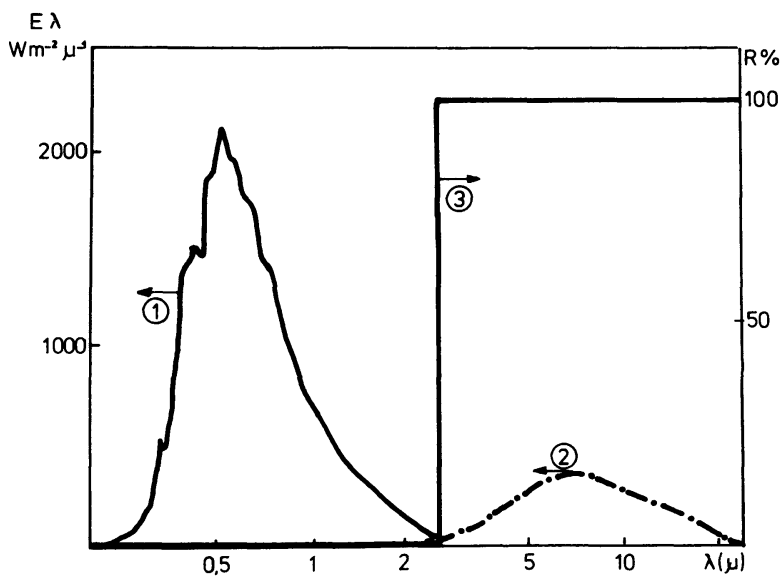

Fig. 1. - 1) Spectre solaire: 2) Spectre émis par le corps noir à $170^{\circ} \mathrm{C}$; 3) Variation du coefficient de réflexion d'une couche sélective idéale.

[1) Solar spectrum; 2) Emission spectrum of a black body at $170^{\circ} \mathrm{C}$; 3) Reflectance of an ideal selective surface.]

Ces différentes surfaces sélectives sont schématisées sur la figure 2. Dans la réalité les choses sont plus complexes et une surface sélective réelle met en jeu plusieurs des phénomènes décrits précédemment.

Malgré la diversité apparente des solutions qui permettent d'obtenir la sélectivité d'une surface, il 

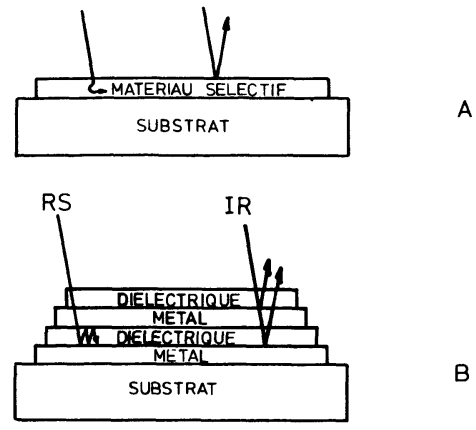

B
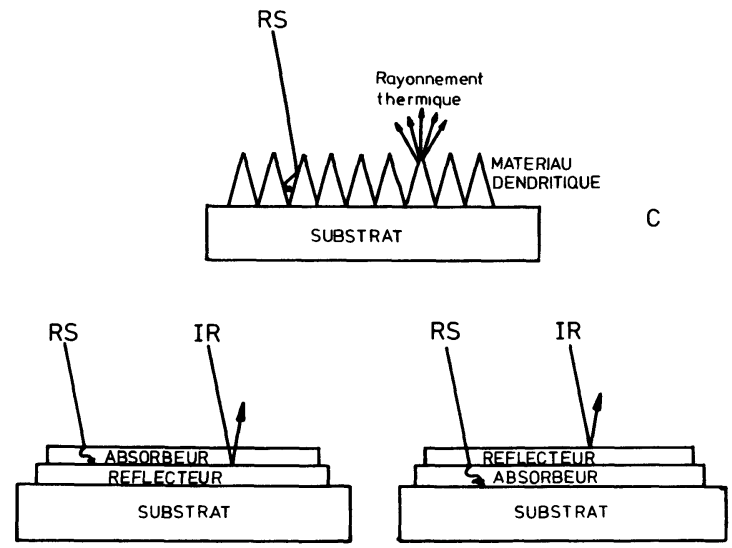

Fig. 2. - Différentes structures de surfaces sélectives.

[Different types of selective surfaces.]

n'y en a que peu qui soient industrialisables, car le matériau doit posséder les propriétés suivantes :

- l'absorptivité solaire " $a_{\mathrm{S}}$ " $\left({ }^{1}\right)$ doit être voisine de 1 , tandis que l'émissivité totale $" e_{\mathrm{T}}$ " $\left({ }^{2}\right)$ doit être aussi faible que possible ;

- les propriétés optiques doivent être stables dans le temps malgré les cyclages thermiques répétés que subira l'absorbeur;

- la surface doit résister à l'agression des agents atmosphériques ;

- enfin, le surcoût dû à la sélectivité doit être compensé par la réduction de la surface de captation nécessaire à une application donnée.

A l'heure actuelle, il semble bien que ce soit le dépôt électrolytique de chrome noir qui remplisse le mieux l'ensemble de ces conditions pour la réalisation de capteurs plans sélectifs.

1. Le dépôt de chrome noir. - Le chrome noir peut être classé dans la catégorie des surfaces sélectives qui sont constituées par l'empilement d'une couche absorbant le rayonnement solaire (la couche de chrome noir proprement dite) sur un substrat réfléchissant le rayonnement infrarouge (cuivre ou nickel).

( ${ }^{1}$ Absorptivité monochromatique hémisphérique moyennée sur le spectre solaire AM1.

$\left({ }^{2}\right)$ Emissivité hémisphérique moyennée sur le spectre du corps noir à la température $T$.
Il en résulte que pour obtenir un effet sélectif marqué, la couche déposée doit satisfaire à deux exigences contradictoires :

- être suffisamment épaisse pour absorber la quasi-totalité du rayonnement solaire;

- être suffisamment mince pour rester transparente au rayonnement infrarouge.

Une épaisseur de 2 à $3000 \AA$ réalise le mieux ce compromis.

Pour illustrer ce phénomène nous avons reporté sur la figure 3 les spectres de réflexion totale hémisphérique de plusieurs échantillons correspondant à des durées croissantes d'électrolyse. Ces échantillons ont été réalisés à l'aide du procédé mis au point par le laboratoire [5].

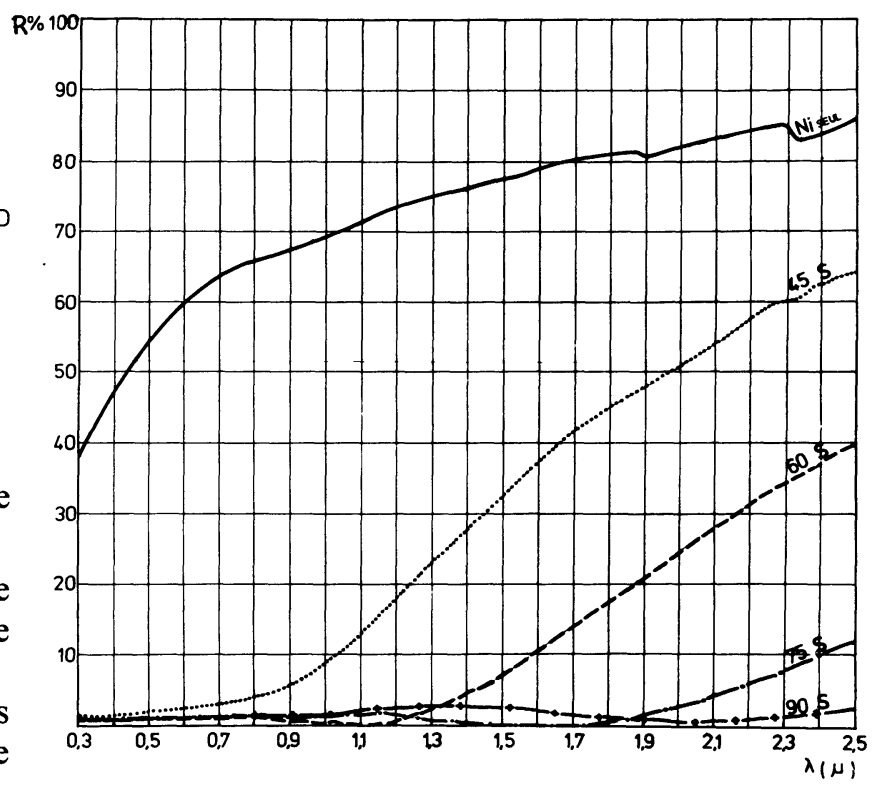

Fig. 3. - Spectre de réflexion totale hémisphérique de la souscouche de nickel et de dépôts de chrome noir de différentes épaisseurs.

[Total hemispherical reflection of the nickel layer and different thicknesses of black chromium.]

Pour $t=0$ on obtient la courbe de la sous-couche de nickel. Puis pour $t=45 \mathrm{~s}$, temps au bout duquel la couche devient continue, la courbe change de forme et l'on observe une absorption marquée dans le visible. Lorsque la durée d'électrolyse augmente, l'épaisseur de la couche croît et les spectres de réflexion se translatent vers les fortes longueurs d'onde. Il en découle donc une augmentation simultanée de l'absorption solaire $\left(a_{\mathrm{s}}\right)$ et de l'émissivité infrarouge $\left(e_{\mathrm{T}}\right)$.

Ces variations respectives, représentées sur la figure 4 , sont toutefois telles que pour une durée d'électrolyse de $65 \mathrm{~s}$, l'absorption solaire atteint 0,97 alors que l'émissivité $\left(e_{170^{\circ} \mathrm{C}}\right)$ n'est que de 0,15 .

Ce procédé de dépôt de chrome noir est développé 


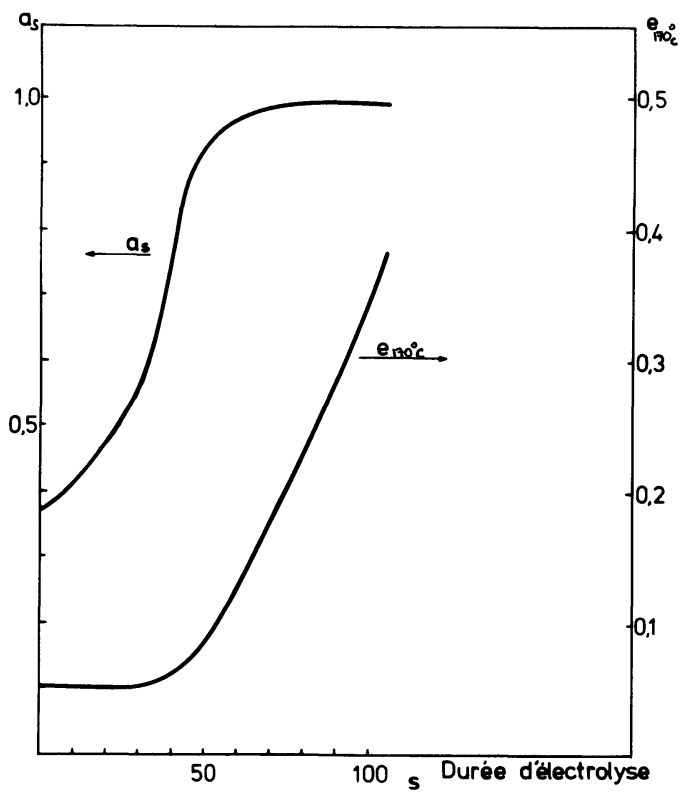

Fig. 4. - Variations de l'absorption et de l'émissivité en fonction de l'épaisseur du dépôt.

[Variation of absorption and emittance as a function of deposited thickness.]

maintenant depuis deux ans à l'échelle industrielle $\left({ }^{1}\right)$ sur des absorbeurs de grandes dimensions (dimensions maximales traitées $3 \times 1,2 \mathrm{~m}$ ) en cuivre et en acier.

La photo $n^{0} 1$ présente une vue générale de la chaîne de traitement dont les différentes séquences sont schématisées sur la figure 5 .

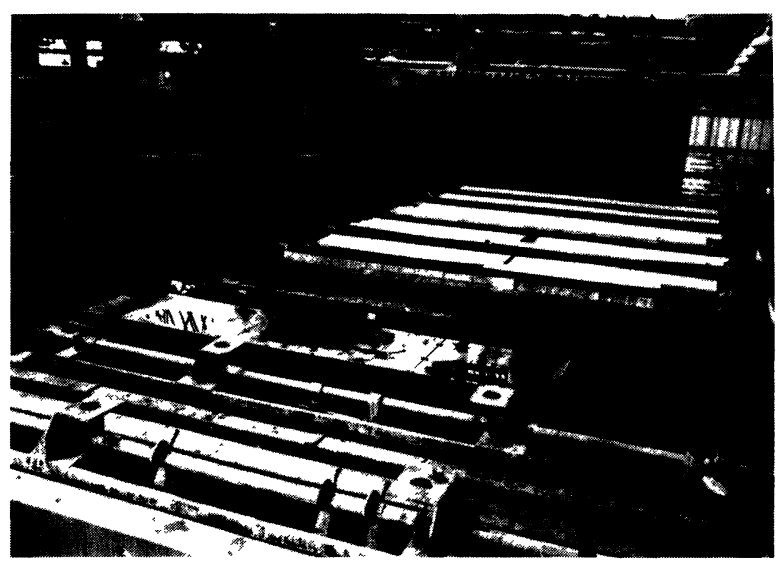

Photo 1. - Vue d'ensemble de la chaîne de traitement industriel de chrome noir (en arrière-plan on remarquera un absorbeur de $2 \mathrm{~m}^{2}$ de surface).

[General view of the production line for black chromium (in the background a $2 \mathrm{~m}^{2}$ absorber can be seen).]

(1) Laminoirs à Froid de Thionville, 16, rue de la Poterne, 60400 Noyon, France.

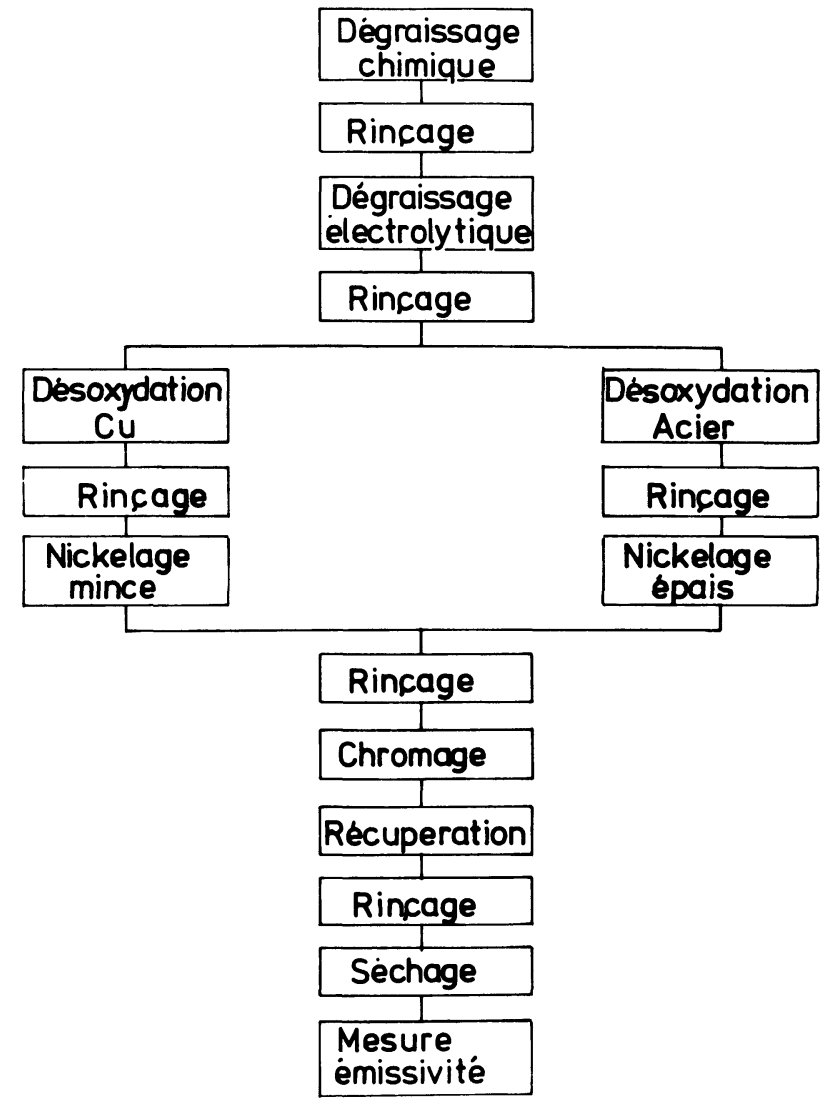

Fig. 5. - Schéma synoptique de la gamme de traitement.

[Schematic of the treatment process.]

Les principaux a vantages du procédé industriel sont les suivants :

- fonctionnement du bain de chrome noir à température ambiante,

- nécessité d'une densité de courant assez faible (inférieure à $15 \mathrm{~A} / \mathrm{dm}^{2}$ ),

- durée d'électrolyse courte (inférieure à $90 \mathrm{~s}$ ),

- conduite aisée du bain : si l'on respecte le rapport de 1,5 entre les surfaces anodique et cathodique, on obtient des dépôts dont $a_{\mathrm{s}}$ est supérieur à 0,95 , tandis que $e_{170^{\circ} \mathrm{C}}$ reste compris entre 0,15 et 0,25 (valeurs mesurées à l'aide d'un appareil de contrôle industriel mis au point par le laboratoire, photo $\mathrm{n}^{\circ} 2$ ), uniquement en faisant des ajouts de $\mathrm{CrO}_{3}$. Ces ajouts périodiques sont déterminés à partir de la mesure de la densité du bain.

2. Caractérisation des couches de chrome noir. A l'œil, le chrome noir présente un aspect velouté qui résulte de la structure superficielle particulière du matériau.

Si on observe la surface de la couche au microscope électronique à balayage (photo $\mathrm{n}^{\circ} 3$ ), on voit qu'elle est constituée par un enchevêtrement de particules allongées. Ces particules, longues de 0,15 à $0,35 \mu$, ont un diamètre compris entre 0,05 et $0,08 \mu$. Une observation au microscope électronique à transmission 


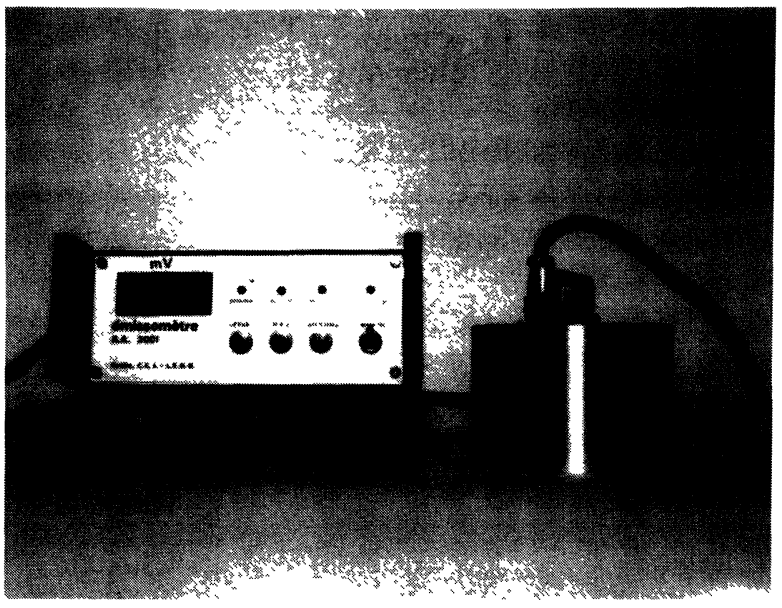

Photo 2. - Emissomètre portatif. Coffret électrique et tête de mesure.

[Portable emissometer : power supply and measuring head.]

faite sur un échantillon préalablement aminci par bombardement ionique révèle par contre trois régions caractéristiques dans le matériau [6] :

a) une région où les aiguilles prédominent. Ces aiguilles sont constituées de grains de chrome métalliques de 40 à $70 \AA$, orientés 111 , noyés dans une phase amorphe d'oxyde de chrome;

b) une région finement cristallisée où l'on retrouve les mêmes grains de chrome métal mais noyés cette fois dans une matrice d'oxydes constituée de $\mathrm{CrO}_{2}-\mathrm{CrO}-\mathrm{Cr}_{3} \mathrm{O}_{4}-\mathrm{Cr}_{2} \mathrm{O}_{3}$;

c) enfin une région complètement amorphe.

Les clichés de diffraction électronique caractéristiques de ces trois régions sont représentés sur la figure 6 .

Le chrome noir déposé par électrolyse est donc un matériau inhomogène ayant une structure voisine de

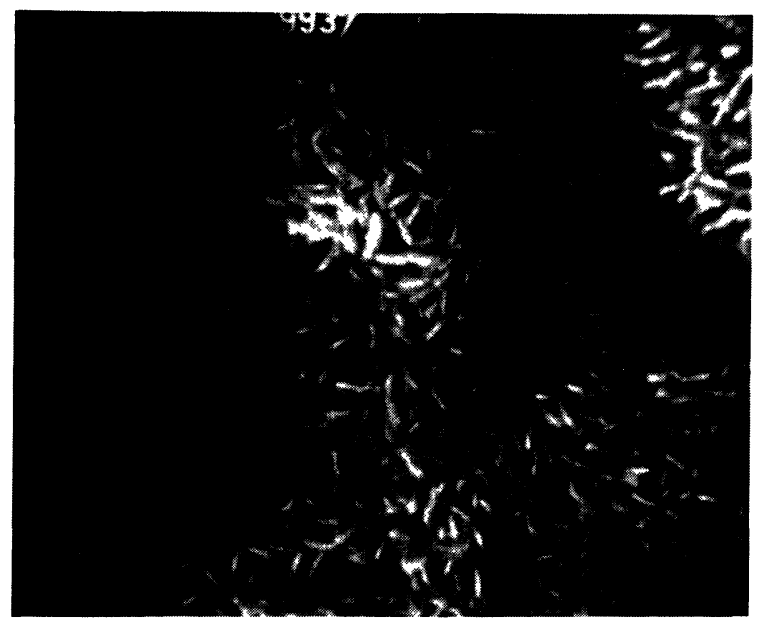

Photo 3. - Photo au microscope à balayage de la couche avant recuit (G 20000 ).

[Scanning electron microscope photograph of the film before annealing $(\times 20000)$.] a)

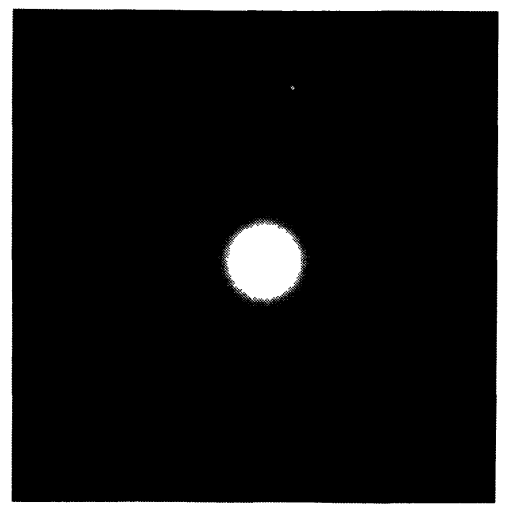

b)

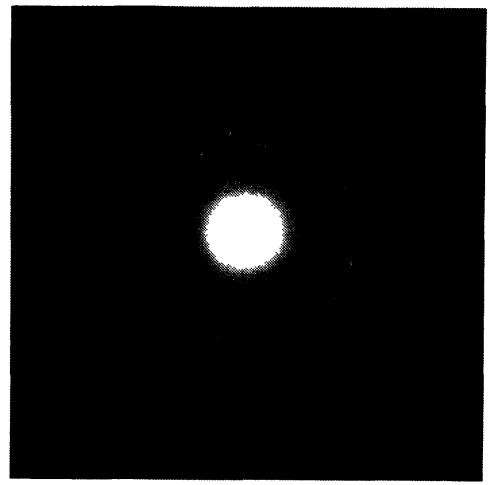

c)

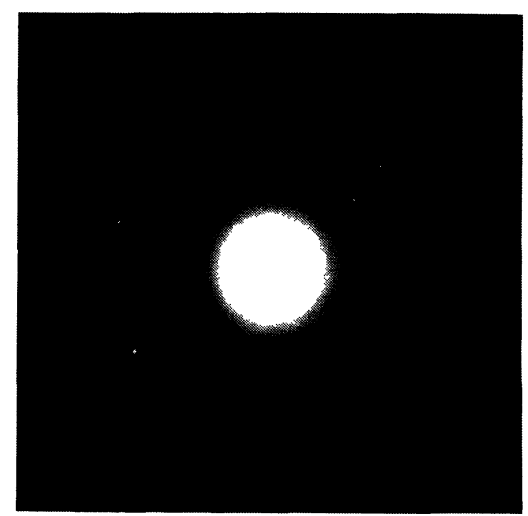

Fig. 6. - Clichés de diffraction des régions a-b-c.

[Electron diffraction patterns of regions a-b-c.]

celle d'un cermet, ce qui lui confère des propriétés optiques particulières.

3. Stabilité thermique. - Pour une bonne fiabilité du capteur, la couche sélective de l'absorbeur doit supporter sans dégradation les nombreux cyclages thermiques auxquels elle sera soumise. Comme des essais préliminaires ont montré que des absorbeurs revêtus de chrome noir pouvaient atteindre des températures de stagnation de $180^{\circ} \mathrm{C}$ dans le cas des capteurs à simple vitrage et de $220^{\circ} \mathrm{C}$ dans le cas du double vitrage, il est nécessaire d'étudier le comportement du matériau en essai de longue durée dans ces zones de température.

Un essai de cyclage thermique de période $1 \mathrm{~h}$ a été 
réalisé entre l'ambiante et $250^{\circ} \mathrm{C}$ sur des échantillons prélevés sur des absorbeurs en cuivre traités industriellement. Au bout de 2000 cycles, il n'a pas été possible de mettre en évidence une évolution des propriétés optiques du matériau.

Par contre, si on simule un fonctionnement continu à plus haute température (cas des capteurs à concentration), on constate qu'à $300^{\circ} \mathrm{C}$ il se produit une légère diminution irréversible de l'absorption et de l'émissivité (Fig. 7). Cette évolution devient beaucoup plus marquée à $350^{\circ} \mathrm{C}$.
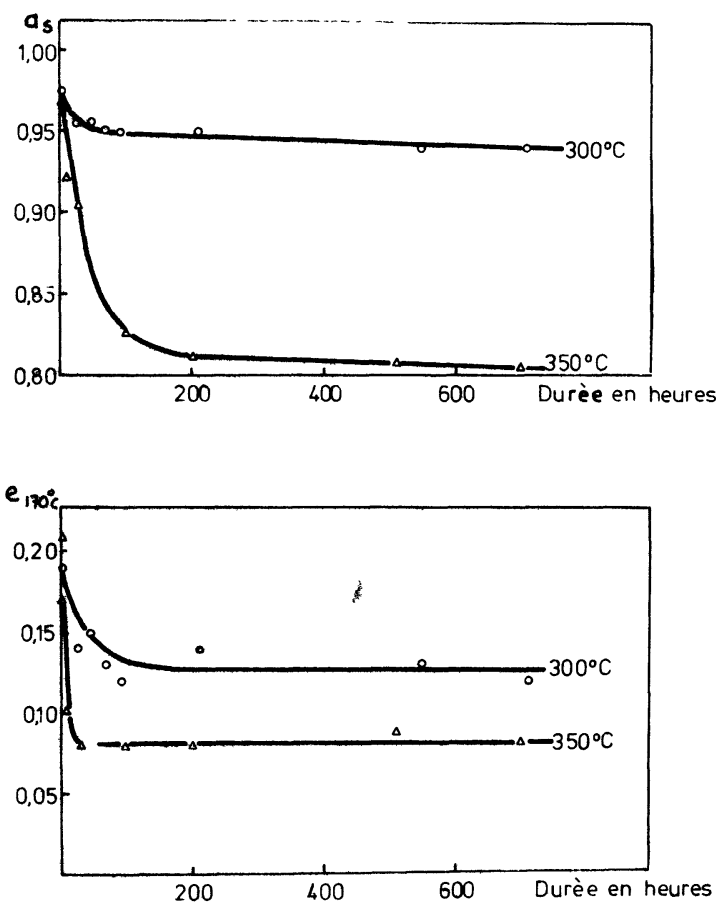

Fig. 7. - Evolution de l'absorption $\left(a_{4}\right)$ et de l'émissivité $\left(e_{170}{ }^{\circ}\right)$ après traitements thermiques à 300 et $350^{\circ} \mathrm{C}$.

[Effect on absorption and emittance of heat treatment at 300 and $350^{\circ} \mathrm{C}$.]

Une étude systématique a été entreprise pour déterminer les mécanismes de la dégradation thermique [6]. Nous n'en résumerons ici que les principales conclusions.

Lorsqu'on observe au microscope à balayage un échantillon de chrome noir recuit à $350^{\circ} \mathrm{C}$ pendant $135 \mathrm{~h}$, temps au bout duquel les propriétés optiques sont pratiquement stabilisées, on constate une profonde modification de la structure (photo $\left.n^{\circ} 4\right)$. En effet, les particules allongées visibles avant recuit sont devenues sphériques, sans doute pour minimiser leur énergie de surface.

Par ailleurs, une étude par diffraction électronique permet de faire les observations suivantes :

- il n'existe plus de régions amorphes,

- la taille des particules de chrome métal reste inchangée mais elles sont maintenant noyées dans une matrice qui ne contient que du $\mathrm{Cr}_{2} \mathrm{O}_{3}$.

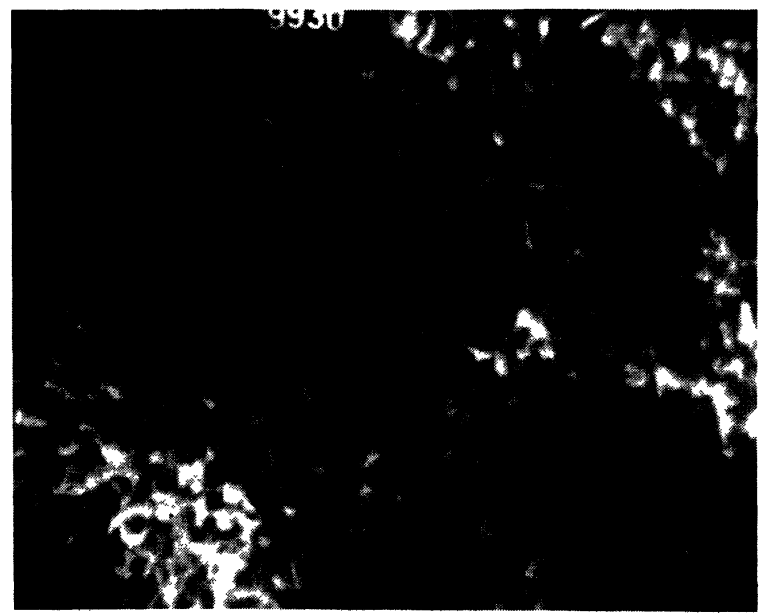

Photo 4. - Photo au microscope à balayage (G 20000$)$ de la couche après un recuit de $135 \mathrm{~h}$ à $350^{\circ} \mathrm{C}$ dans l'air.

[Scanning electron microscope photograph of the film after annealing for $135 \mathrm{~h}$ at $350^{\circ} \mathrm{C}$ in air $(\times 20000)$.]

Le recuit à haute température provoque donc à la fois une modification physico-chimique et une modification structurale de la couche.

Pour expliquer ce phénomène, nous avons réalisé un modèle à partir de la théorie de Maxwell Garnet modifiée par Cohen [7] et de la théorie de McKenzie et McPhedran [8].

Avant traitement thermique, on assimile les particules allongées à des ellipsoïdes ayant un rapport axial de 4 (valeur déduite des observations faites au microscope électronique à balayage). Pour que le spectre de réflexion calculé soit en accord avec le spectre expérimental, on doit aussi introduire un gradient dans la fraction volumique de l'oxyde en fonction de l'épaisseur (courbes 1, Fig. 9) : la fraction volumique maximale a été prise égale à celle qui correspond aux particules en contact et la fraction volumique minimale, à l'interface air-couche, a été prise égale à zéro.

$\mathrm{Au}$ cours du recuit on observe une modification de la forme des particules. Si dans le modèle on remplace les ellipsoïdes par des sphères on obtient alors, toutes conditions égales par ailleurs, une modification notable du spectre de réflexion (courbe 2, Fig. 9), qui reste en bon accord avec la courbe expérimentale obtenue après un traitement thermique de $24 \mathrm{~h}$ (courbe 2, Fig. 8).

Enfin, si dans le modèle on tient compte de l'homogénéisation du matériau [9] et de la densification qui en résulte, on obtient la courbe 3 de la figure 9 qui présente un maximum vers $0,7 \mu$. Un maximum situé à la même longueur d'onde était aussi présent sur la courbe expérimentale au bout d'un traitement de $135 \mathrm{~h}$ (courbe 3, Fig. 8).

Ce déplacement des spectres vers les courtes longueurs d'onde au cours du recuit est dû à une diminution de l'épaisseur optique qui se traduit par une augmentation de la réflectivité, surtout dans l'infra- 


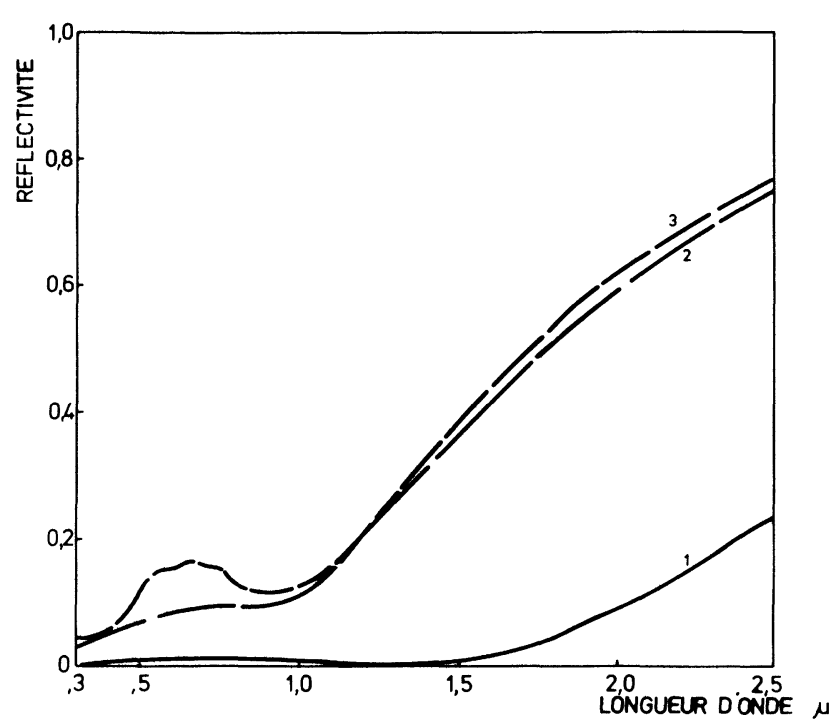

Fig. 8. - Courbes expérimentales du coefficient de réflexion hémisphérique en fonction de la longueur d'onde : 1) avant recuit; 2) après recuit $24 \mathrm{~h}$ à $350^{\circ} \mathrm{C} ; 3$ ) après recuit $135 \mathrm{~h}$ à $350^{\circ} \mathrm{C}$.

[Experimental reflexion spectra as a function of wavelength : 1) before annealing ; 2) after annealing for $24 \mathrm{~h}$ at $350^{\circ} \mathrm{C} ; 3$ ) after annealing for $135 \mathrm{~h}$ at $350^{\circ} \mathrm{C}$.]

rouge. Il en résulte donc une diminution irréversible de $a_{\mathrm{s}}$ et $e_{\mathrm{T}}$ au cours du traitement thermique.

Cette interprétation du mécanisme de la dégradation thermique où l'on montre que le facteur prépondérant est le changement de la forme des particules, ce qui nécessite donc un transport de matière, nous a conduit à penser que pour une température donnée il y avait un seuil de durée en dessous duquel des cyclages thermiques, même répétés, ne provoquaient pas de modifications irréversibles.

Pour vérifier cette hypothèse nous avons donc réalisé une nouvelle série de cyclages thermiques entre l'ambiante et $350^{\circ} \mathrm{C}$, mais en ne laissant l'échantillon que $5 \mathrm{~min}$ à la température maximale. Le résultat actuel portant sur 50 cycles montre qu'il n'y a pas de variation significative des propriétés optiques, alors que pour une durée équivalente de traitement continu l'absorption avait déjà diminué.

Cette constatation permet donc d'envisager l'emploi du chrome noir dans certaines applications où le matériau pourrait être porté de manière accidentelle

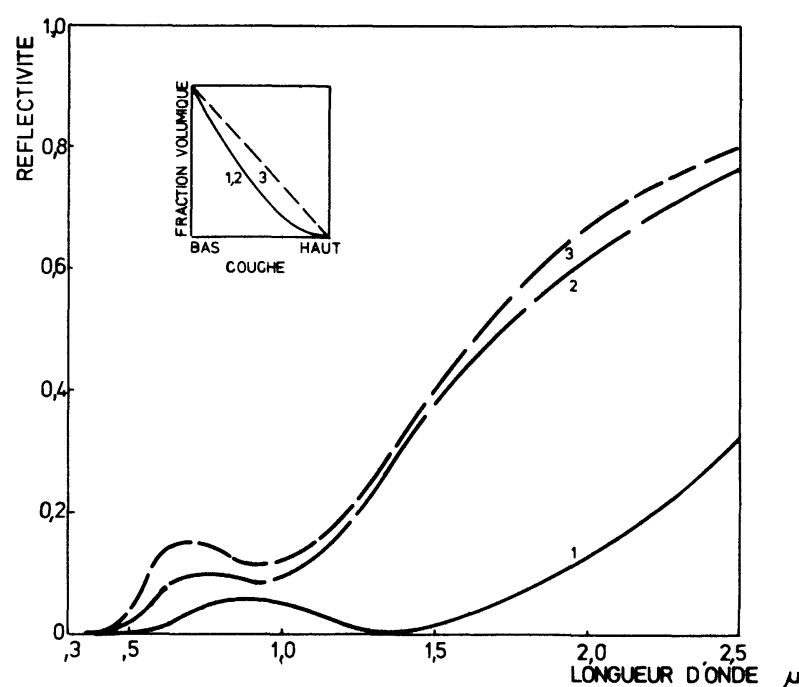

Fig. 9. - Courbes du coefficient de réflexion obtenues avec le modèle : 1) particules ellipsoïdes ; 2) particules sphériques ; 3) film plus homogène. Les courbes de l'encart indiquent la variation de la fraction volumique dans les 3 cas considérés.

[Reflection spectra as a function of wavelength with the model : 1) ellipsoidal particles; 2) spherical particles; 3) uniform film.]

durant des temps très brefs à des températures bien supérieures à celle de son utilisation en continu.

4. Conclusion. - Le procédé de dépôt de chrome noir qui a été mis au point par le laboratoire permet actuellement de traiter de manière industrielle des absorbeurs de grandes dimensions. En raison de la stabilité thermique de la couche obtenue, le matériau est particulièrement adapté au revêtement des capteurs plans équipés d'une ou deux vitres.

Pour l'emploi dans les capteurs à concentration il faudra prendre de grandes précautions car un fonctionnement continu à $300^{\circ} \mathrm{C}$ commence à dégrader de manière irréversible les propriétés optiques de la couche sélective.

Remerciements. - Nous tenons à remercier M. Tran Van Danh pour la réalisation des échantillons, ainsi que MM. Lombard, Basq et Sharma pour l'étude et l'interprétation des clichés de microscope électronique.

\section{Bibliographie}

[1] TABOR, H., Trans. Conf. on the use of Solar Energy 1955, vol. 2 (University of Arizona Press, Arizona) 1958, p. 41.

[2] Mattox, D. M., J. Vac. Sci. Techn. 13 (1976) 127.

[3] Seraphin, B. O. et Meinel, A. B., Photothermal energy conversion and the Optical properties. B. O. Seraphin (ed.) Optical properties of solids New Developments (North Holland, Amsterdam) 1976.

[4] Spitz, J., Aubert, A., Behaghel, J. M., Berthier, S., Lafait, J., Rrvory, J., Rerue Phys. Appl. 14 (1979) 67.
[5] Spitz, J., Tran Van Danh, Aubert, A., Sol. Energy Mater. 1 (1979) 189

[6] Ritchie, I. T., Sharma, S. K., SPitz, J., Valignat, J. (à paraître dans Sol. Energy Mater.).

[7] Cohen, R. W., Cody, G. D., Couts, M. D., Abeles, B., Phys. Rev. B 8 (1973) 3689.

[8] McKenzie, D. R., McPhedran, Nature 265 (1977) 128, and Proc. R. Soc. London A 359 (1978) 45.

[9] Ritchie, I. T., Window, B., Appl. Opt. 16 (1977) 1438. 